\title{
DYNAMICAL SYMMETRY GROUP $O(4,2)$ OF HYDROGEN ATOM ENERGY SPECTRUM AND WEIGHT DIAGRAM OF THE GROUP IRREDUCIBLE REPRESENTATION
}

\author{
Igor Ya. Doskoch \\ Lebedev Physical Institute, Russian Academy of Sciences \\ Leninskii Prospect 53, Moscow 119991, Russia \\ E-mail: iggor@ sci.lebedev.ru
}

Keywords: weight diagram, hydrogen-atom energy spectrum, foundations of quantum mechanics.

In this comment, we describe graphically the irreducible representation of the dynamical symmetry group $O(4,2)$, which contains complete information on the wave function (state vector $|n \ell m\rangle$ ) and discrete energy levels of hydrogen atom as well as yields natural connection of the representation with all quantum numbers $(n=1,2, \ldots, \ell=0,1, \ldots n-1, m=-\ell,-\ell+1, \ldots, \ell)$ of the hydrogen-atom states.

Fock found the $O(4)$ compact symmetry group (called the hidden symmetry group) of the Schrödinger equation for hydrogen atom that explains the quantum degeneracy of discrete energy levels of hydrogen atom $E_{n}=-1 / 2 n^{2}$ (in dimensionless units) with respect to the orbital quantum number $\ell$, in addition to the natural rotation symmetry $O(3)$ of the Coulomb potential $-1 / r=U(r)$, explaining the degeneracy of the energy levels with respect to the magnetic quantum number $m$.

In [1] was shown that all the states of discrete spectrum of hydrogen atom (or corresponding solutions of the Schrödinger equation) realize an

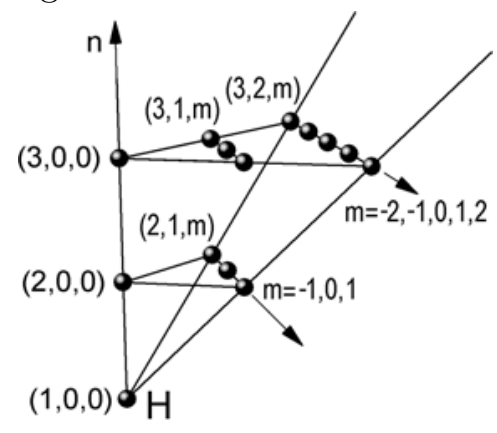
infinite-dimension irreducible representation of noncompact symmetry group of hydrogen atom $O(4,2)$. The finite-dimension representations of the hidden symmetry describe the states belonging to the $n$th energy levels. The degeneracy of these levels is equal to $n^{2}$. So the ground state $(n=1)$ is not degenerated, the first excited state $(n=2)$ has the degeneracy of the energy level equal to 4 , the second excited state $(n=3)$ has degeneracy 9 , and so on.

All the discrete levels are united as basis vectors of irreducible representation of $O(4,2)$. The history of this result can be found in books [2,3], review article [4]; it is developed in [5,6]. In this comment, we describe the weight diagram of the discrete energy levels of hydrogen atom by a triangle pyramid, being naturally connected with quantum numbers $n, \ell$, and $m$. Since $\sum_{\ell=1}^{n} \sum_{m=-\ell}^{\ell} 1=n^{2}$, these states of thee $n$th energy levels can be presented not only as squares [7] but also as triangles, as shown in the figure.

\section{References}

1. I. A. Malkin and V. I. Man'ko, JETP Lett., 2, 146 (1965).

2. R. Gilmore, Lie Groups, Lie Algebras 85 Some of Their Applications (Dover Books on Mathematics), Dover, Mineola, NY (2005).

3. A. Barut, A. Bohm, and Y. Neeman, Dynamical Groups and Spectrum Generating Algebras, World Scientific, Singapore (1986).

4. M. R. Kibler, Found. Chem., 9, 221 (2007).

5. A. O. Barut and H. Kleinert. Phys. Rev., 156, 1541 (1967).

6. G. Mack and I. Todorov, J. Math. Phys., 10, 2078 (1969).

7. M. A. Man'ko and V. I. Man'ko, J. Russ. Laser Res., 40, 6 (2019).

Manuscript submitted by the author in English on December 6, 2018. 